\title{
¿Existen diferencias en América Latina en el aporte de la educación preescolar al logro educativo futuro?: PISA 2009-2012
}

\author{
Luis Fernando Gamboa y Natalia Krüger
}

RESUMEN

\begin{abstract}
En este artículo se provee de evidencia sobre la existencia de diferencias en el rendimiento en pruebas académicas en la enseñanza media entre quienes asistieron al nivel inicial y quienes no lo hicieron. A partir de datos del Programa para la Evaluación Internacional de Alumnos (PISA) de 2009 a 2012 respecto de varios países latinoamericanos, se utiliza un método no paramétrico basado en la generación de contrafactuales que descompone las brechas entre factores observables y no observables. Se encuentra segregación por nivel socioeconómico en el acceso; brechas de puntajes considerables —condicionales a los controles utilizados - y más amplias mientras más tiempo se permanece en educación inicial; y diferencias importantes en las brechas entre países. En general, las brechas halladas son mayores en Lectura que en Matemáticas.
\end{abstract} Educación (ICFEs).1fgamboa@gmail.com

Natalia Krüger es Investigadora Asistente del Instituto de Investigaciones Económicas y Sociales del Sur (Consejo Nacional de Investigaciones Científicas y Técnicas, conicet) y Asistente de Docencia del Departamento de Economía de la Universidad Nacional del Sur, Argentina. natalia.kruger@uns.edu.ar 


\section{I}

\section{Introducción}

El cuidado integral en la primera infancia, reconocido como un derecho del niño y una inversión social clave, es promovido actualmente por múltiples organismos, acuerdos y normas internacionales. Los compromisos asumidos en la Declaración Mundial sobre la Educación para todos —adoptada en Jomtien en 1990- y en la definición de los objetivos de la educación para todos —realizada en Dakar durante el año 2000_, revelan la existencia de un consenso en torno de la importancia que reviste la educación durante los primeros años de vida.

Este período se considera crucial en el desarrollo físico, mental y emocional de los sujetos (CEPAL, 2008; SITEAL, 2010), de modo que garantizar una atención de calidad para todos los niños resulta esencial en términos de la justicia social. Asimismo, desde diversas disciplinas se han destacado los efectos positivos de la educación infantil en las posteriores trayectorias educativas, a través de la formación de habilidades cognitivas y nocognitivas (MEC, 2013). Según Heckman (2000), estos efectos determinan altas tasas de retorno para la inversión en la educación temprana. Las intervenciones en la niñez permitirían a su vez ahorrar recursos en el futuro en el área social, facilitarían la inserción laboral de las mujeres y fomentarían el desarrollo socioeconómico a nivel global.

La educación inicial se vincula estrechamente con la equidad social, tanto por su valor intrínseco como instrumental ${ }^{1}$. El acceso desigual representa una vulneración de los derechos de los niños, especialmente en contextos heterogéneos, al tiempo que constituye una fuente de inequidad futura debido a la repercusión de esta instancia en una amplia gama de resultados socioeconómicos.

En América Latina coexisten escenarios muy diferentes en términos de la oferta y la demanda de educación preescolar, que responden a la gran diversidad de contextos socioeconómicos, culturales e institucionales entre y dentro de los países. A su vez, no está claro en qué medida la existencia de desigualdades en el acceso puede atentar contra la igualdad de oportunidades educativas a lo largo de la vida, ya que es escasa la literatura a nivel

\footnotetext{
${ }^{1}$ Este nivel recibe nombres diferentes en los distintos países en función de sus objetivos curriculares e institucionales (Diker, 2003). A lo largo del presente trabajo se utilizarán indistintamente denominaciones como educación "preescolar", "inicial" o "en la primera infancia".
}

regional en que se estudia empíricamente la influencia de la escolarización temprana en los aprendizajes posteriores.

Por ello, en este documento se procura ilustrar el grado de equidad en el acceso al nivel preescolar y evaluar la manera en que afecta al logro educativo de mediano plazo. La estrategia consiste en cuantificar las brechas de rendimiento en el nivel secundario entre los grupos de alumnos que asistieron previamente al nivel inicial y quienes no lo hicieron, condicionales a una serie de variables de control. Se intenta no solo conocer la magnitud de estas brechas en los países latinoamericanos, sino también realizar comparaciones entre ellos.

Los logros se aproximan mediante los resultados obtenidos por los estudiantes en las pruebas del Programa para la Evaluación Internacional de Alumnos (PISA, por sus siglas en inglés), administrado por la Organización para la Cooperación y el Desarrollo Económicos (OCDE). Se utiliza la información correspondiente a las rondas 2009 y 2012 de los siete países latinoamericanos participantes en ambas: Argentina, Brasil, Chile, Colombia, México, Perú y Uruguay ${ }^{2}$.

Para identificar los beneficios de haber asistido al nivel inicial se emplea un método no paramétrico ideado por Ñopo (2008), que permite comparar grupos de alumnos muy similares en sus características personales y contextuales. El método provee una estimación de la brecha de desempeño - diferencial promedio de puntaje en cada área- entre los estudiantes que asistieron al nivel preescolar y quienes no lo hicieron, descomponiéndola entre factores observables y no observables. Así, es posible identificar qué parte de la brecha "bruta" puede atribuirse al efecto de otras variables asociadas tanto al acceso al nivel inicial, como al rendimiento en las pruebas académicas, obteniendo una brecha "neta" o libre de estas influencias. En comparación con el método tradicional de descomposición de Blinder-Oaxaca, esta descomposición tiene la ventaja de evitar los posibles sesgos debidos a las diferencias en los soportes de las distribuciones de las características para ambos grupos.

Los análisis realizados indican que existen brechas de rendimiento considerables, aun controlando por un

\footnotetext{
2 Si bien Costa Rica también ha participado en el programa, en el primer caso lo hizo en un operativo posterior realizado en el año 2010, por lo que se decidió excluirlo del presente análisis para asegurar una estricta comparabilidad intertemporal.
} 
completo conjunto de factores asociados al entorno familiar y escolar de los estudiantes. Es decir, los resultados sugieren que la educación inicial genera un diferencial relevante en el logro educativo a los 15 años de edad. Además, se encuentra que una asistencia más prolongada al preescolar reporta mayores beneficios en el futuro.

La estructura del trabajo es la siguiente: luego de esta Introducción, en la sección II se describe la situación actual y la evolución reciente de la cobertura de la educación inicial en América Latina; en la sección III se resumen los antecedentes sobre el impacto de asistir al preescolar en los logros educativos posteriores; la metodología se detalla en la sección IV, y en la sección V se describen los datos y las variables seleccionadas. En la sección VI se presentan los resultados y, finalmente, en la sección VII se recogen las conclusiones del estudio.

\section{II}

\section{La asistencia al nivel educativo inicial en}

\section{América Latina: metas y avances}

Las Metas Educativas 2021, aprobadas por la Conferencia Iberoamericana de Educación y refrendadas en el año 2010, incluyen ambiciosos objetivos vinculados con la atención de la primera infancia, reflejando la relevancia política que el tema ha adquirido últimamente en la región. Sin embargo, como afirma Poggi en SITEAL (2013), el creciente interés proviene de varias décadas atrás.

Históricamente, la atención temprana se ha provisto de manera dispersa, principalmente a través de programas privados de carácter asistencial. Ya en las décadas de 1960 y 1970 comenzaron a elaborarse regulaciones y se redoblaron los esfuerzos por expandir la educación inicial desde el Estado, reconociendo en mayor medida su función pedagógica (Diker, 2003). La implementación de programas integrales y sostenidos en dicho período ha permitido que se registren considerables avances en el acceso, respondiendo en parte a la presión de demanda generada por la incorporación de la mujer al mercado laboral.

No obstante, solo a partir de los años noventa se generalizó en América Latina la meta de universalización de al menos el último año del preescolar (Albergucci, 2006), lo que se tradujo en una significativa expansión de la oferta y de la matrícula. En general, se ha establecido la obligatoriedad de la asistencia a una institución educativa a partir de los 5 años de edad — como es el caso de Colombia-, si bien en algunos países el ciclo obligatorio comprende a los niños de 4 años — como en la Argentina, el Uruguay y el Brasil- e incluso a los de 3 años: México y el Perú. En Chile, por su parte, aún no se ha legislado en este sentido, siendo la educación obligatoria solo a partir del nivel primario (SITEAL, 2009). En general, las normas en relación con la obligatoriedad evidencian que la atención continúa concentrada en las edades más próximas al ingreso a la educación primaria, quedando relegada la población de niños de 0 a 3 años (MEC, 2013).

Estas iniciativas han contribuido al incremento constante de la cobertura educativa para el grupo etario de 3 a 5 años. Según la información provista por la Base de Datos Socioeconómicos para América Latina y el Caribe (SEDLAC), a principios de la presente década aproximadamente el $70 \%$ de los niños de esa edad asistían a una institución educativa en los siete países considerados, con un cierto rezago en el caso de Colombia (52\%). En 20 años, se ha producido una expansión de la asistencia cercana al $10 \%$ en México, por ejemplo, pero mayor que el $100 \%$ en países como la Argentina y Chile.

La actual diversidad de programas formales e informales, así como la ausencia de información, restringen la implementación de políticas de calidad en educación preescolar. Frecuentemente, las condiciones en que se desarrollan las propuestas en términos de recursos físicos, humanos y pedagógicos, derivan en resultados limitados y de escasa pertinencia (Cordero, 2004; SITEAL, 2009). De hecho, existe evidencia acerca de la insuficiente atracción de capital humano altamente calificado hacia este sector, lo que responde en gran medida a la baja remuneración que perciben los docentes de nivel preescolar (Mizala y Ñopo, 2012).

Además de lo anterior, en la región persisten importantes desigualdades en el acceso a este nivel según el contexto socioeconómico de pertenencia y el área de residencia. En el cuadro 1 se presentan las tasas de asistencia para distintos grupos de niños de 5 años de edad alrededor del año 2000 -momento aproximado en el que los alumnos participantes de PISA 2009 o 2012 tenían dicha edad - y alrededor de 2011. 
Tasas brutas de escolarización según factores relacionados.

Población de niños de 5 años de edad, 2000 y 2011

(En porcentajes)

\begin{tabular}{|c|c|c|c|c|c|c|c|c|c|c|}
\hline & \multicolumn{2}{|c|}{ Género } & \multicolumn{3}{|c|}{ Clima educativo del hogar ${ }^{\mathrm{a}}$} & \multicolumn{3}{|c|}{$\begin{array}{l}\text { Nivel de ingresos per cápita } \\
\text { familiares }^{b}\end{array}$} & \multicolumn{2}{|c|}{ Área } \\
\hline & Mujer & Varón & Bajo & Medio & Alto & Bajo & Medio & Alto & Rural & Urbana \\
\hline \multicolumn{11}{|c|}{ Alrededor de 2000} \\
\hline Argentina & 73,6 & 73,8 & 64,3 & 72,9 & 81,3 & 70,8 & 80,6 & 86,9 & & 73,7 \\
\hline Brasil & 66,9 & 65,0 & 57,4 & 70,3 & 85,3 & 62,1 & 74,9 & 87,9 & 48,3 & 70,1 \\
\hline Chile & 70,6 & 73,0 & 52,3 & 69,1 & 82,0 & 70,9 & 76,9 & 86,3 & 45,1 & 76,4 \\
\hline Colombia & 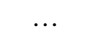 & $\ldots$ & $\ldots$ & $\ldots$ & & & & & $\ldots$ & $\ldots$ \\
\hline México & 85,0 & 85,5 & 71,0 & 91,0 & 97,8 & 83,1 & 93,4 & 93,3 & 76,6 & 88,6 \\
\hline Perú & 70,6 & 79,4 & 61,2 & 80,2 & 93,4 & 74,6 & 78,1 & 94,3 & 66,1 & 80,9 \\
\hline$\underline{\text { Uruguay }}$ & 91,9 & 91,8 & 89,0 & 91,1 & 97,1 & 90,0 & 92,5 & 99,0 & $\ldots$ & 91,9 \\
\hline \multicolumn{11}{|c|}{ Alrededor de 2011} \\
\hline Argentina & 93,5 & 93,7 & 76,3 & 93,1 & 97,1 & 92,0 & 94,9 & 98,5 & $\ldots$ & 93,6 \\
\hline Brasil & 87,3 & 85,9 & 80,7 & 88,7 & 94,5 & 84,8 & 91,4 & 95,4 & 78,8 & 88,3 \\
\hline Chile & 86,9 & 87,0 & 89,7 & 94,1 & 94,3 & 93,1 & 97,7 & 95,2 & 88,3 & 94,8 \\
\hline Colombia & 92,8 & 94,9 & 79,6 & 89,3 & 98,6 & 89,9 & 90,0 & 93,7 & 73,4 & 90,7 \\
\hline México & 97,3 & 98,6 & 89,8 & 97,5 & 99,7 & 95,4 & 98,6 & 99,5 & 93,9 & 97,3 \\
\hline Perú & 91,0 & 92,0 & 80,6 & 92,8 & 99,0 & 94,2 & 94,7 & 99,4 & 79,5 & 95,7 \\
\hline Uruguay & 96,8 & 95,8 & 93,3 & 96,6 & 96,3 & 95,4 & 96,1 & 97,4 & 98,3 & 96,0 \\
\hline
\end{tabular}

Fuente: Sistema de Información de Tendencias Educativas en América Latina (SITEAL), sobre la base de las respectivas Encuestas Nacionales de Hogares.

a Promedio de años de educación de los miembros de 18 años o más: Bajo para menos de 6 años; Medio para 6 a 12 años, y Alto para 12 años o más.

b El nivel Bajo incluye al 30\% de los hogares con menores ingresos; el Medio a hogares entre el percentil 30 y el 60 , y el Alto al $40 \%$ de los hogares con mayores ingresos.

En ambos momentos el capital cultural de las familias, representado aquí por el clima educativo de los hogares, es un determinante clave de la inclusión de los niños en este nivel. Si bien se han registrado avances desde inicios del presente siglo, actualmente existen brechas relevantes en la asistencia según el nivel educativo de las familias, especialmente en los casos de la Argentina, Colombia y el Perú. Una tendencia similar, aunque más atenuada, se visualiza en relación con el nivel de ingresos per cápita familiares, registrándose en el Brasil las brechas más importantes.

El lugar de residencia ha constituido históricamente un factor significativo, ya que los niños de 5 años que habitan en zonas rurales asisten en menor medida a una institución escolar. Esto se debe principalmente a la insuficiente oferta educativa en muchas de estas localidades, aunque también puede relacionarse con factores socioeconómicos y culturales de las familias. Si bien ha mejorado la cobertura en el área rural durante el período, el acceso continúa siendo diferenciado, sobre todo en Colombia, el Perú y el Brasil.

Por otra parte, no parece existir un sesgo de género en la asistencia al preescolar, ya que las brechas entre varones y mujeres son reducidas, sin inclinarse en favor de uno u otro grupo.

En síntesis, durante las últimas décadas se observa una expansión de la cobertura del nivel inicial impulsada por el marco legal en América Latina. Sin embargo, más allá de los objetivos normativos, la existencia de segregación en el acceso pone de manifiesto que el alcance de las leyes y compromisos se encuentra limitado por las desigualdades sociales y económicas vigentes. En la actualidad, al menos, puede resaltarse una deuda o desafío en tres sentidos: i) incorporar a la población de niños más pequeños (de 0 a 4 años); ii) incrementar la inclusión de aquellos provenientes de sectores sociales vulnerables y de los residentes de zonas rurales, y iii) promover y evaluar la calidad de los servicios ofrecidos. 


\section{III}

\section{Evidencia reciente y revisión de la literatura}

El acceso a la educación temprana es un derecho fundamental del niño, pero también es un medio para iniciar su vinculación con el sistema escolar. Durante los primeros años de vida se desarrollan las principales capacidades cognitivas y no-cognitivas que afectan el desempeño en la escuela (Glewwe y Jacoby, 1995; Burchinal y otros, 1997; Currie, 2001; Berhman, Cheng y Todd, 2004; Barnett y Lamy, 2006; Cunha y otros, 2006; Nores y Barnett, 2010; Skibbe y otros, 2011; y Hazarika y Viren, 2013). Los efectos pueden ser heterogéneos en el corto y el largo plazo, como consecuencia de la varianza en la calidad de los programas. Por ende, el principal obstáculo para analizar los tipos de efectos generados por la asistencia al preescolar radica en la información sobre su calidad ${ }^{3}$.

La influencia de la educación inicial se ha abordado en la literatura mediante diferentes metodologías. Empleando metaanálisis para resumir estos resultados, Camilli y otros (2010) encuentran que la educación preescolar produce un efecto inmediato de aproximadamente 0,50 desviaciones estándar en el desarrollo cognitivo, que es equivalente a moverse del percentil 30 al 50 en pruebas

\footnotetext{
${ }^{3}$ La información disponible para evaluar la importancia de cada uno de estos aspectos no es frecuente en países latinoamericanos, como sí lo es en países desarrollados como los Estados Unidos de América: programas State pre-K (Preescolar Estatal), Head Start (ventaja inicial) o Preschool Special Education (Programa Especial de Educación Preescolar). Allí ha sido posible seguir a cohortes completas y con ello controlar por factores no observables.
}

\section{IV}

\section{Metodología}

La metodología empleada en el presente documento sigue el enfoque no paramétrico utilizado por Nopo (2008). Dicho método, además de permitir la cuantificación de la brecha existente en términos de la variable de resultado (por ejemplo, puntaje en la prueba PISA), provee una descomposición de acuerdo con las características de la población de estudio que se compara. Dado que no se estandarizadas de logros. En materia social y emocional, los efectos encontrados son menores, pero significativos (0,33 desviaciones estándar). Este tipo de hallazgos brinda soporte a las afirmaciones de Heckman (2000 y 2008), quien menciona que la mayor ganancia en las inversiones educativas se produce cuando se llevan a cabo en los primeros años de vida.

Un ejemplo notorio en la literatura es realizado por Arteaga y otros (2014), por medio de datos de una cohorte de 1.500 estudiantes del Estudio Longitudinal de Chicago matriculados en 1980. Los autores utilizan puntuación de propensión (propensity score weighting) y encuentran que quienes asisten por dos años al preescolar tienen menos probabilidades de recibir atención escolar especial o de ser vinculados con el crimen, con respecto a quienes hacen solo un año de preescolar. Estos resultados alimentan la evidencia en favor de los efectos de largo plazo de haber tenido una asistencia más larga al preescolar. Por su parte, Magnuson, Ruhm y Waldfogelet (2007a y 2007b), utilizando datos del Early Chilhood Longitudinal Study, analizan el efecto de la asistencia al nivel inicial en la habilidad de los niños en la escuela, y encuentran que quienes cursan esos programas tienen mayores habilidades en Lectura y Matemáticas al ingresar al colegio (0,18-0,12 desviaciones) - las que tienden a decrecer durante el primer año-, pero al mismo tiempo presentan una propensión a tener problemas de comportamiento, la que sí persiste por algún tiempo después del ingreso al colegio. Sin embargo, los autores no reportan evidencia de efecto causal, por lo que la conclusión debería ser tomada con cautela.

dispone de un diseño experimental o cuasi experimental, se adopta una metodología basada en la generación de contrafactuales para reducir los posibles sesgos, pero que exige cautela en la interpretación. Existen factores observables y no observables (como la calidad de los programas de preescolar o la movilidad entre planteles durante la vida académica) que podrían influir en los 
resultados. El uso del método mencionado reduce el sesgo proveniente de factores no observables, pero no posee el grado de alcance que tendría un ejercicio experimental con una muestra aleatoria, de manera que no se puede identificar un efecto causal en sentido estricto. A diferencia de las comparaciones de tratamiento y control (PSM, por sus siglas en inglés), este enfoque hace un emparejamiento de individuos basado en características y no en puntajes.

Sea $Y$ una variable que representa el puntaje obtenido por un estudiante en una prueba académica, en función de un vector de características $X$. El puntaje esperado por un alumno, condicional a sus características y al hecho de haber asistido al preescolar $(P)$, estaría dado por $E[Y \mid P, X]=g^{P}(x)$. Por su parte, el puntaje esperado para quienes no asistieron $(N P)$ será $E[Y \mid N P, X]=g^{N P}(x)$. Así, el diferencial de rendimiento entre quienes asistieron y quienes no asistieron al nivel inicial (brecha de puntaje) estará dado por la ecuación (1):

$$
\Delta=E[Y \mid P, X]-E[Y \mid N P, X]
$$

Asumiendo que $F^{P}(\cdot)$ y $F^{N P}(\cdot)$ representan las funciones de distribución condicional de las características individuales $\mathrm{X}$ y que son funciones que van del espacio vectorial $\mathrm{R}^{\mathrm{n}}$ a $\mathrm{R}$, es posible definir la medida de probabilidad sobre el soporte de la distribución $\mathrm{S}$ en $d F^{P}(\cdot)$ y $d F^{N P}(\cdot)$ como:

$$
\begin{gathered}
\mu^{P}(S)=\int_{S} d F^{P}(x) \\
\mu^{N P}(S)=\int_{S} d F^{N P}(x)
\end{gathered}
$$

Teniendo en cuenta las dos expresiones anteriores, al reemplazar en la ecuación (1) se tendrá:

$$
\Delta=\int_{S^{P}} g^{P}(x) d F^{P}(x)-\int_{S^{N P}} g^{N P}(x) d F^{N P}(x)
$$

El principal reto para la obtención del diferencial insesgado del puntaje proviene del hecho de que el soporte de las distribuciones de características puede ser distinto ${ }^{4}$ $\mathrm{y}$, por lo tanto, es necesario hacerlo comparable. Para

\footnotetext{
${ }^{4}$ Es decir, podría haber individuos que asistieron al preescolar que tienen valores en alguna característica $\mathrm{X}$, como el nivel de estudios de sus padres, que no pueden hallarse en el grupo de individuos que no asistieron al preescolar, y viceversa.
}

ello, Ñopo (2008) propone descomponer cada integral en dos partes: una reúne la intersección de los soportes (soporte común) y otra está fuera de ese soporte. Este paso consiste en remplazar a $S^{P}$ por $S^{P} \cap S^{N P}$ y $\overline{S^{N P}} \cap S^{P}$ lo mismo para $S^{N P}$.

$$
\begin{gathered}
\Delta=\left[\int_{S^{N P} \cap S^{P}} g^{P}(x) d F^{P}(x)+\int_{S^{N P} \cap S^{P}} g^{P}(x) d F^{P}(x)\right] \\
-\left[\int_{S^{N P} \cap S^{P}} g^{N P}(x) d F^{N P}(x)+\int_{S^{N P} \cap S^{P}} g^{N P}(x) d F^{N P}(x)\right]
\end{gathered}
$$

Además, dado que los valores de $d F^{P}(\cdot)$ y $d F^{N P}(\cdot)$ son por definición nulos fuera del soporte, el dominio de las integrales se extiende a $\overline{S^{P}}$ y $\overline{S^{N P}}$. Al reemplazar $\mu^{N P}\left(S^{P}\right)$ por $1-\mu^{N P}\left(\overline{S^{P}}\right)$ y $\mu^{P}\left(S^{N P}\right)$ por $1-\mu^{P}\left(\overline{S^{N P}}\right)$, se tiene una expresión que permite descomponer la brecha total. El paso final consiste en agregar y quitar el elemento necesario para poder evaluar el contrafactual, es decir, el puntaje que habría obtenido el estudiante que no asistió a la educación inicial si en realidad hubiese asistido.

De esta manera, la expresión total quedará:

$$
\begin{aligned}
& \Delta=\left[\int_{S^{N P}} g^{P}(x) \frac{d F^{P}(x)}{\mu^{P}\left(\overline{S^{N P}}\right)}-\int_{S^{N P}} g^{P}(x) \frac{d F^{P}(x)}{\mu^{P}\left(S^{N P}\right)}\right] \mu^{P}\left(S^{N P}\right) \\
& +\int_{S^{P} \cap S^{N P}} g^{P}(x)\left[\frac{d F^{P}(x)}{\mu^{N P}\left(S^{N P}\right)}-\frac{d F^{N P}(x)}{\mu^{N P}\left(S^{P}\right)}\right](x) \\
& +\int_{S^{P} \cap S^{N P}}\left[g^{P}(x)-g^{N P}(x)\right] \frac{d F^{N P}(x)}{\mu^{N P}\left(S^{P}\right)} \\
& +\left[\int_{S^{P}} g^{N P}(x) \frac{d F^{N P}(x)}{\mu^{N P}\left(S^{P}\right)}-\int_{S^{P}} g^{N P}(x) \frac{d F^{N P}(x)}{\mu^{N P}\left(\overline{S^{P}}\right)}\right] \mu^{P}\left(\overline{S^{N P}}\right)
\end{aligned}
$$

Teniendo en cuenta cada uno de estos términos, se tendrá que:

$$
\Delta_{P}=\left[\int_{S^{N P}} g^{P}(x) \frac{d F^{P}(x)}{\mu^{P}\left(\overline{S^{N P}}\right)}-\int_{S^{N P}} g^{P}(x) \frac{d F^{P}(x)}{\mu^{P}\left(S^{N P}\right)}\right] \mu^{P}\left(S^{N P}\right)
$$


$\Delta_{P}$ es la parte de la brecha que proviene de diferencias en las características de dos grupos que han asistido al preescolar: quienes están dentro y fuera del soporte común. Es decir, aquellos cuyas características pueden ser emparejadas con las de los $N P$, y aquellos que tienen características que no pueden serlo.

El segundo término, $\Delta_{x}$, es la parte de la brecha total entre los que asistieron y no asistieron, que puede ser explicada por diferencias en la distribución de las características de la población que queda dentro del soporte común. Formalmente, esta fracción será:

$$
\Delta_{X}=\int_{S^{P} \cap S^{N P}} g^{P}(x)\left[\frac{d F^{P}(x)}{\mu^{N P}\left(S^{N P}\right)}-\frac{d F^{N P}(x)}{\mu^{N P}\left(S^{P}\right)}\right](x)
$$

El tercer término de la ecuación (4), $\Delta_{0}$, también se define sobre el soporte común —en el que se encuentran individuos con características similares provenientes de las dos poblaciones comparadas-y es el de mayor interés en este estudio. Como en el caso de la tradicional descomposición Blinder-Oaxaca, $\Delta_{0}$ (definido en la ecuación (7)), es la fracción de la brecha en el puntaje que no puede ser explicada por las diferencias en las características observables entre los individuos que asistieron y no asistieron al preescolar. Es decir, es el diferencial en el puntaje que persiste cuando se hace una comparación de los dos grupos, $P$ y $N P$, teniendo en cuenta a aquellos que poseen valores muy similares en una serie de atributos $X$. Por lo tanto, puede atribuirse al efecto propio de haber asistido al nivel inicial y a posibles factores inobservables remanentes (niveles de esfuerzo, factores genéticos, estado físico, movilidad entre planteles, calidad de la educación recibida y otros).

$$
\Delta_{0}=\int_{S^{P} \cap S^{N P}}\left[g^{P}(x)-g^{N P}(x)\right] \frac{d F^{N P}(x)}{\mu^{N P}\left(S^{P}\right)}
$$

El último término, $\Delta_{N P}$, explica las diferencias entre ambos grupos de las características de quienes no asistieron a la educación inicial: aquellos que pueden ser emparejados con los que asisten y aquellos que no pueden serlo.

${ }^{5} \Delta_{p}$ sería cero (0) si todos los estudiantes que asistieron al preescolar pudieran ser emparejados con quienes no asistieron. Para mayor detalle sobre este método, véase Ñopo (2008).

$$
\Delta_{N P}=\left[\int_{S^{P}} g^{N P}(x) \frac{d F^{N P}(x)}{\mu^{N P}\left(S^{P}\right)}-\int_{\overline{S^{P}}} g^{N P}(x) \frac{d F^{N P}(x)}{\mu^{N P}\left(\overline{S^{P}}\right)}\right] \mu^{P}\left(\overline{S^{N P}}\right)(8)
$$

Teniendo en cuenta estas definiciones, y reordenando los términos para representar la parte que es explicada por el método y la parte que proviene de aspectos no observables, la brecha agregada o bruta queda de la siguiente manera:

$$
\Delta=\left(\Delta_{P}+\Delta_{X}+\Delta_{N P}\right)+\Delta_{0}
$$

En el procedimiento empírico para la obtención de esta descomposición se utiliza un algoritmo de emparejamiento que se lleva a cabo en cinco pasos. Este algoritmo está pensado para lograr la comparación de individuos lo más parecidos posible en cuanto a sus características observables, pero que a la vez difieren en si han asistido o no a la educación inicial.

En primer lugar, se selecciona aleatoriamente un individuo participante de la prueba PISA dentro del grupo que declara haber atendido al preescolar $(P)$ a partir del cual se pretende llevar a cabo la comparación. Luego, se seleccionan todos aquellos estudiantes que no asistieron al preescolar y que son similares al individuo escogido en el primer paso, es decir, que comparten con aquel una determinada característica. En tercer lugar, se crea un individuo sintético $(N P)$ a partir de la muestra de individuos que no asistieron al preescolar, con un desempeño equivalente al promedio obtenido por todos los individuos de dicha muestra en la asignatura de análisis de la prueba PISA (Matemáticas o Lectura). Teniendo un individuo con preescolar y otro sin preescolar con condiciones similares, ambos se emparejan. Finalmente, se repite el proceso para el resto de los individuos que hicieron el preescolar, de manera que para cada uno de ellos se tendrá un individuo sintético con el que se lo compara. Este último paso se hace con reemplazamiento, evitando sesgos provenientes del ordenamiento. A partir de la muestra emparejada, se halla la diferencia promedio del valor esperado en el puntaje y se obtiene la brecha de resultados.

La principal ventaja de esta metodología con respecto a la tradicional descomposición de Blinder-Oaxaca (Blinder, 1973; Oaxaca, 1973), es que contempla las diferencias en los soportes de las distribuciones de los atributos $X$, evitando posibles sesgos en la estimación de las brechas entre ambos grupos. A su vez, esta estimación no está condicionada a una forma funcional específica, con los requisitos que ello impone sobre las variables. 
Sin embargo, también es necesario advertir sobre una limitación del método. Por una parte, la posibilidad de comparar entre quienes asisten y quienes no asisten al nivel inicial será más precisa en la medida en que el conjunto de variables empleadas para hacer el emparejamiento sea más amplio. Por otra, si se incorpora un gran número de atributos $X$, el tamaño muestral implicaría que el conjunto de estudiantes utilizado para la creación del individuo sintético sería más pequeño y podría ser menos confiable. Es decir, que existe un trade-off entre ambos objetivos, que determina la necesidad de escoger con cuidado las variables de control, seleccionando un conjunto reducido pero de alta relevancia. De cualquier modo, esta limitación implica que no se puede hablar directamente de un efecto causal de la educación preescolar. Con el fin de tener mayor certeza de que los resultados no están influenciados por sesgos muestrales, se realizó un ejercicio de simulación para evaluar la significancia del tamaño de las brechas ${ }^{6}$.

${ }^{6}$ El ejercicio de ténica de bootstrap se realizó extrayendo 200 submuestras aleatorias al $95 \%$ de tamaño de la muestra original.

\section{Datos y variables}

La prueba PISA evalúa alumnos de 15 años que asisten al séptimo grado o a uno superior. La muestra del programa surge de un proceso en dos etapas: se obtiene inicialmente una muestra estratificada de escuelas, y luego se selecciona al azar a un grupo de alumnos en cada una de ellas. Este diseño garantiza la representatividad de la muestra en términos de la población objetivo a nivel país, si bien el nivel de cobertura es variable. Para los países latinoamericanos participantes, la muestra de alumnos —una vez ponderada por los pesos correspondientescubre desde el 58,5\% de la población total de jóvenes de 15 años, en el caso de Colombia en el año 2009, hasta el $85,2 \%$, en el caso de Chile (OCDE, 2012 y 2014).

En el operativo realizado cada tres años desde el año 2000 se evalúan las competencias en Matemáticas, Ciencias y Lectura, enfatizando una de ellas cada año en forma rotativa. Además, se aplican cuestionarios complementarios a alumnos y directivos de las escuelas sobre el contexto personal y familiar de los estudiantes y respecto de las características de los centros (Adams y Wu, 2002).

Estos datos permiten construir la variable Preescolar, que indica si el alumno: i) nunca asistió al nivel inicial; ii) asistió durante 1 año o menos; iii) asistió durante más de un año. Como puede apreciarse, en la muestra existen diferencias entre los países en términos del acceso al preescolar por parte de los participantes del estudio, encontrándose la Argentina, México y el Uruguay en la mejor situación (véase el cuadro 2$)^{7}$.

En la medición de la brecha de rendimiento se emplean los "valores plausibles" reportados en PISA, que representan el rango de habilidades que puede tener un estudiante en las distintas competencias y son comparables entre países y años. Se escogieron las rondas de 2009 y 2012 del estudio, porque la información de la asistencia al nivel inicial no está disponible para las versiones previas. A su vez, se consideraron las áreas de Matemáticas y Lectura, porque en ellas se concentran dichas ondas del programa.

Al comparar los resultados obtenidos en las pruebas PISA según las categorías de la variable Preescolar sin ningún control, se advierte una relación positiva entre la asistencia al nivel inicial - y su duración - y el desempeño en el nivel secundario en todos los países (véase el cuadro 3). El incremento porcentual en el puntaje obtenido en las pruebas para quienes asistieron más de un año al preescolar en comparación con quienes no asistieron es generalmente superior en la Argentina, el Uruguay y el Perú, tanto en 2009 como en 2012.

\footnotetext{
${ }^{7}$ Dado que PISA solo evalúa a los jóvenes que logran continuar sus estudios hasta el nivel secundario y con un nivel relativamente bajo de retraso, los resultados en esta muestra sobrestiman la tasa de asistencia real en la población total.
} 
Descripción de la variable preescolar, 2009 y 2012

(En porcentajes)

\begin{tabular}{|c|c|c|c|c|c|c|c|c|}
\hline & \multicolumn{4}{|c|}{2009} & \multicolumn{4}{|c|}{2012} \\
\hline & \multicolumn{3}{|c|}{ Valores válidos } & \multirow{2}{*}{$\begin{array}{l}\text { Valores } \\
\text { perdidos }\end{array}$} & \multicolumn{3}{|c|}{ Valores válidos } & \multirow{2}{*}{$\begin{array}{l}\text { Valores } \\
\text { perdidos }\end{array}$} \\
\hline & No asistió & $\begin{array}{l}\text { Asistencia } \\
\leq 1 \text { año }\end{array}$ & $\begin{array}{l}\text { Asistencia } \\
>1 \text { año }\end{array}$ & & No asistió & $\begin{array}{l}\text { Asistencia } \\
\leq 1 \text { año }\end{array}$ & $\begin{array}{l}\text { Asistencia } \\
>1 \text { año }\end{array}$ & \\
\hline Argentina & 4,7 & 29,1 & 66,2 & 2,6 & 6,2 & 22,6 & 71,2 & 2,3 \\
\hline Brasil & 21,3 & 33,4 & 45,3 & 6,8 & 19,1 & 33,6 & 47,3 & 3,5 \\
\hline Chile & 15,0 & 52,8 & 33,2 & 3,5 & 9,2 & 56,5 & 34,3 & 2,3 \\
\hline Colombia & 18,5 & 53,3 & 28,2 & 1,6 & 14,2 & 52,4 & 33,3 & 1,7 \\
\hline México & 10,3 & 19,5 & 70,2 & 1,5 & 9,5 & 18,7 & 71,8 & 1,1 \\
\hline Perú & 15,1 & 26,3 & 58,6 & 7,3 & 13,8 & 25,0 & 61,2 & 2,9 \\
\hline Uruguay & 12,8 & 15,9 & 71,3 & 2,9 & 16,2 & 14,1 & 66,7 & 4,3 \\
\hline Total países & 15,7 & 31,7 & 52,6 & 4,4 & 14,1 & 31,0 & 54,9 & 2,5 \\
\hline
\end{tabular}

Fuente: Elaboración propia sobre la base de Organización para la Cooperación y el Desarrollo Económicos (OCDE), PISA 2009 Technical Report, París, OECD Publishing, 2012; y PISA 2012 Results: What Students Know and Can Do: Student Performance in Mathematics, Reading and Science (Volume 1), París, OECD Publishing, 2014.

CUADRO 3

\section{Puntaje promedio en las pruebas PISA, total y según categorías de la variable preescolar, 2009 y 2012}

\begin{tabular}{|c|c|c|c|c|c|c|c|}
\hline & Argentina & Brasil & Chile & Colombia & México & Perú & Uruguay \\
\hline \multicolumn{8}{|c|}{2009} \\
\hline \multicolumn{8}{|l|}{ Matemáticas } \\
\hline Total & $388,1(0,74)$ & $385,8(0,82)$ & $421,1(0,69)$ & $380,8(0,82)$ & $418,5(0,42)$ & $365,1(1,27)$ & $426,7(1,07)$ \\
\hline No asistió & $334,7(2,21)$ & $358,0(0,93)$ & $392,9(1,56)$ & $349,2(1,23)$ & $375,1(0,91)$ & $335,7(2,03)$ & $373,1(2,13)$ \\
\hline Asistencia $\leq 1$ año & $369,6(0,87)$ & $386,4(1,13)$ & $419,9(0,83)$ & $386,1(1,42)$ & $416,2(0,82)$ & $363,7(2,12)$ & $410,8(2,05)$ \\
\hline Asistencia > 1 año & $403,3(1,06)$ & $407,8(0,97)$ & $441,2(1,23)$ & $394,5(1,02)$ & $426,9(0,33)$ & $383,5(1,06)$ & $442,8(1,15)$ \\
\hline \multicolumn{8}{|l|}{ Lectura } \\
\hline Total & $398,3(0,99)$ & $411,7(0,55)$ & $449,4(0,83)$ & $413,2(0,55)$ & $425,3(0,65)$ & $369,7(0,95)$ & $425,8(0,61)$ \\
\hline No asistió & $331,5(4,02)$ & $378,9(0,78)$ & $418,5(1,50)$ & $380,1(1,04)$ & $378,2(1,24)$ & $336,9(1,78)$ & $368,4(1,33)$ \\
\hline Asistencia $\leq 1$ año & $379,1(1,41)$ & $414,1(1,16)$ & $452,4(1,19)$ & $418,8(0,87)$ & $424,2(0,91)$ & $367,8(1,41)$ & $404,7(1,23)$ \\
\hline Asistencia $>1$ año & $415,7(1,21)$ & $438,7(0,57)$ & $465,3(1,02)$ & $428,7(1,36)$ & $434,2(0,55)$ & $391,5(0,98)$ & $444,5(0,85)$ \\
\hline \multicolumn{8}{|c|}{2012} \\
\hline \multicolumn{8}{|l|}{ Matemáticas } \\
\hline Total & $388,4(1,16)$ & $388,5(0,66)$ & $422,6(0,69)$ & $376,5(0,40)$ & $413,3(0,33)$ & $358,1(0,71)$ & $409,3(0,45)$ \\
\hline No asistió & $337,2(2,25)$ & $365,6(0,74)$ & $381,4(2,54)$ & $350,5(1,01)$ & $378,0(0,99)$ & $327,5(1,60)$ & $369,7(0,73)$ \\
\hline Asistencia $\leq 1$ año & $365,8(1,44)$ & $382,7(0,69)$ & $422,6(0,76)$ & $379,5(0,94)$ & $411,2(0,45)$ & $360,4(1,46)$ & $389,9(1,07)$ \\
\hline Asistencia > 1 año & $402,8(1,28)$ & $404,9(0,91)$ & $435,7(0,90)$ & $384,8(1,08)$ & $419,3(0,38)$ & $383,6(0,81)$ & $426,3(0,77)$ \\
\hline \multicolumn{8}{|l|}{ Lectura } \\
\hline Total & $395,9(1,24)$ & $406,5(0,62)$ & $441,4(0,71)$ & $403,4(0,44)$ & $423,5(0,42)$ & $384,1(1,11)$ & $411,3(0,55)$ \\
\hline No asistió & $336,7(3,11)$ & $378,9(1,04)$ & $401,4(2,97)$ & $374,4(1,63)$ & $383,3(1,27)$ & $342,1(2,07)$ & $367,7(1,82)$ \\
\hline Asistencia $\leq 1$ año & $372,7(1,94)$ & $400,9(0,83)$ & $442,6(0,69)$ & $406,6(0,30)$ & $422,4(0,68)$ & $374,4(2,14)$ & $396,1(1,46)$ \\
\hline Asistencia > 1 año & $411,9(1,26)$ & $425,9(0,88)$ & $452,5(1,10)$ & $413,5(1,16)$ & $430,2(0,48)$ & $401,8(1,11)$ & $430,2(0,56)$ \\
\hline
\end{tabular}

Fuente: Elaboración propia sobre la base de Organización para la Cooperación y el Desarrollo Económicos (OCDE), PISA 2009 Technical Report, París, OECD Publishing, 2012; y PISA 2012 Results: What Students Know and Can Do: Student Performance in Mathematics, Reading and Science (Volume 1), París, OECD Publishing, 2014.

Nota: Las diferencias de medias entre las categorías "Asistencia $\leq 1$ año" y "No asistió", y entre "Asistencia $>1$ año" y "No asistió" resultan estadísticamente significativas al nivel del $1 \%$ en todos los casos; entre paréntesis se presentan los errores estándar de las estimaciones. 
En el extremo opuesto se encuentran Chile, en 2009, y Colombia, en 2012, como los países con menores diferencias promedio.

Teniendo en cuenta que las brechas dependen de factores personales y contextuales vinculados simultáneamente a las posibilidades de acceso al preescolar y al rendimiento académico en la escuela media (que en general están fuera del control del individuo), se utilizan los siguientes factores de control en el proceso de emparejamiento ${ }^{8,9}$ :

- Características individuales:

Familia nuclear: esta variable (igual a uno si el alumno convive con ambos padres, y a cero en otro caso) puede estar relacionada con los logros cognitivos (Calero y otros, 2007; Krüger, 2013) y no-cognitivos (Krüger, Formichella, Lekuona, 2015). Asimismo, en varios de los países considerados se observa una mayor probabilidad de asistencia al nivel inicial para aquellos alumnos provenientes de una familia nuclear ${ }^{10}$.

Además, para controlar por el nivel socioeconómico (NSE) de las familias se incluye el índice de estatus económico, social y cultural elaborado por el equipo de PISA. En este se sintetiza información relativa a tres dimensiones principales del hogar: el máximo nivel educativo alcanzado por ambos padres; la máxima jerarquía ocupacional; y el acceso a posesiones de riqueza, culturales y educativas (OCDE, 2012). Todos ellos son factores que se relacionan significativamente con las posibilidades de éxito educativo (Krüger, 2013), al tiempo que parecen ser determinantes relevantes del acceso y la permanencia en el nivel preescolar.

\footnotetext{
8 Si bien el género suele considerarse un posible determinante de los logros educativos, se omite porque en estimaciones previas se encuentra que no explica los diferenciales de acceso al preescolar.

${ }^{9}$ La descripción está disponible a solicitud.

${ }^{10}$ La base de datos incluye observaciones con información incompleta (missing data) que, para el caso de los controles utilizados, tiende a concentrarse en la variable Familia nuclear. No obstante esto, al realizar varias simulaciones con y sin esta variable, se encuentran resultados estadísticamente equivalentes. Estos ejercicios pueden ser solicitados a los autores.
}

- Características de las escuelas:

Escuela pública: los alumnos de escuelas privadas en general presentan un mejor desempeño en las pruebas estandarizadas. Sin embargo, no está claro que esta situación pueda atribuirse específicamente al tipo de gestión, ya que parece más bien responder a las características de la población estudiantil que recibe cada tipo de escuela (Formichella y Krüger, 2013). Su inclusión obedece a que representa una amplia gama de factores escolares y familiares que podrían incidir en el rendimiento de los alumnos y, al mismo tiempo, relacionarse significativamente con su trayectoria educativa previa.

Localización urbana: en general, el acceso al preescolar suele estar vinculado al área de residencia, como se verifica en la muestra de PISA. Asimismo, es una variable proxy de la disponibilidad de recursos físicos $\mathrm{y}$ humanos, que suele ser mayor y de mejor calidad en las zonas urbanas.

Puntaje promedio de los compañeros de la escuela: con esta última variable se procura capturar las diferencias de calidad que pueden existir entre las escuelas medias. Ante la posibilidad de que aquellas familias que deciden -o pueden - enviar a sus hijos al nivel inicial logren luego enviarlos a mejores centros de nivel secundario, se hace necesario controlar por este factor para no atribuir erróneamente su efecto a la asistencia al preescolar. El nivel de calidad de la escuela a la que asiste cada alumno se aproxima a través del puntaje promedio obtenido en la prueba PISA de Matemáticas o Lectura por parte de sus compañeros de escuela. Dicho puntaje reflejaría tanto la influencia del nivel académico de los compañeros, como la correlación de los recursos y las prácticas escolares con el rendimiento propio. Para los efectos de la metodología, esta variable se presenta en quintiles.

Cabe mencionar dos limitaciones que surgen de la utilización de esta base de datos. En primer lugar, no hay información disponible sobre el tipo de colegio donde se cursó la educación inicial y primaria. En segundo lugar, existe un problema de selección, ya que están excluidos de la muestra aquellos alumnos que, habiendo asistido o no al nivel inicial, abandonaron sus estudios antes de los 15 años, o se encuentran cursando un grado inferior al séptimo. Si la asistencia al preescolar incide también en la progresión o culminación de los estudios, la evaluación de las brechas en los puntajes PISA estaría subestimando el efecto total en los logros educativos, y este es un punto que se quiere hacer explícito. 


\section{V}

\section{Resultados}

La muestra de PISA refleja que los jóvenes de 15 años de la región han accedido de manera diferenciada al nivel inicial en función de su contexto socioeconómico de pertenencia (suponiendo que en la actualidad este es similar al vigente en su infancia). Así, es claro que quienes asistieron más de un año al preescolar pertenecen más probablemente a una familia de alto nivel socioeconómico, seguidos por aquellos que asistieron durante un año o menos. De igual modo, la asistencia al preescolar y su duración se asocian positivamente con la residencia en una localidad urbana y con la asistencian en el nivel medio a una escuela privada, de mayor calidad, o de ambas características según lo indican los logros de los compañeros.

Estas influencias se reflejan luego en la reducción del tamaño del soporte común y en el crecimiento del componente explicado de la brecha $\left(\Delta_{x}\right)$ a medida que se agregan las variables de emparejamiento. El análisis del efecto individual de cada control muestra que el puntaje promedio de los pares explica un porcentaje superior al $50 \%$ de la brecha, siendo este impacto mayor en 2012; por su parte, la inclusión del nivel socioeconómico (NSE) familiar explica más de un $30 \%$. Luego, la gestión escolar explicaría por sí sola entre el $15 \%$ y el $30 \%$ de las brechas, con la excepción de México. La localización es un factor significativo en el Perú, Colombia y México, pero de menor relevancia en el resto de la región. Por último, la estructura familiar es el factor menos influyente, en general responsable por menos de un $10 \%$ de la diferencia inicial, aunque su repercusión aumentó considerablemente en $2012^{11}$.

Los coeficientes de mayor interés son las brechas brutas de desempeño $(\Delta$, ecuación (1)) y la fracción que permanece sin explicación $\left(\Delta_{0}\right.$, ecuación (7)) luego de los controles utilizados (véanse los cuadros 4 y 5). La lectura de las estimaciones en cada área tiene dos componentes: el primero refleja la brecha bruta de puntajes - diferencia promedio entre los grupos-; el segundo componente incluye los controles que se inician con la localización y luego incorporan sucesivamente los demás factores. Así, la brecha promedio en Lectura para el agregado de países entre quienes asistieron menos de un año y quienes no lo hicieron es de $9,9 \%$ y 7,8\% para 2009 y 2012 ,

\footnotetext{
${ }^{11}$ Estos cálculos pueden ser solicitados a los autores.
}

respectivamente, quedando finalmente sin explicar una diferencia de $3,5 \%$ y $2,3 \%$ (coeficiente "+Nota media pares" de los cuadros 4 y 5 , penúltima fila).

Las brechas brutas estimadas son notorias en todos los países: los estudiantes que asistieron al nivel inicial obtuvieron resultados en PISA que en promedio superaron a los de quienes no asistieron entre un $4 \%$ y un $26 \%$ según el año, el país y la cantidad de años de asistencia al preescolar (mayor exposición a experiencias educativas). Estas brechas son mayores mientras más tiempo estuvieron los estudiantes expuestos a la educación preescolar.

Después de controlar por todas las características familiares y escolares, las brechas no explicadas continúan siendo positivas y, en muchos casos, sustanciales. Considerando a los siete países en su conjunto, la brecha máxima se da en Lectura $(4,2 \%)$ cuando se compara entre asistir más de un año al preescolar y no asistir en 2009; y la mínima para Matemáticas (1,9\%) entre asistir un año o menos al preescolar y no hacerlo en 2012. A nivel individual, estas brechas $\left(\Delta_{0}\right.$, ecuación (7)) oscilan entre un $0,1 \%$ y un $11 \%$ del puntaje en favor de quienes asistieron al nivel preescolar.

La fracción de la brecha bruta que persiste sin explicación al considerar todos los controles $\left(\Delta_{0} / \Delta\right)$ es en promedio del $30 \%$. Esta razón es menor en el caso de la asistencia al preescolar durante más de un año, lo que refleja que los factores socioeconómicos considerados son más significativos como determinantes del acceso cuando la asistencia supera a la instancia obligatoria.

Por otra parte, se aprecian diferencias relevantes entre los países tanto en la magnitud inicial de las brechas como después del emparejamiento. Al contemplar los diferenciales brutos de resultados, la Argentina es en general el país donde se observa la mayor ventaja por parte de aquellos que accedieron a una educación temprana. En 2009, se ubican luego el Uruguay o México, presentando Chile las brechas más bajas. En 2012, el Uruguay y Chile le siguen a la Argentina con las brechas más altas, correspondiendo las mínimas a Colombia o el Brasil. Es decir que, con la excepción de la Argentina, no se registra un patrón uniforme al comparar entre países las diferencias brutas.

Por su parte, la brecha neta o no explicada por las variables de control es más alta en la Argentina y el Uruguay en los dos períodos. Por ejemplo, un 10,6\% de la diferencia en puntaje en Lectura entre quienes asisten 
CUADRO 4

Descomposición de las brechas de puntajes en las pruebas PISA, 2009

(En porcentajes)

"Asistió más de 1 año" comparado con "No asistió"

\begin{tabular}{|c|c|c|c|c|c|c|c|c|}
\hline & Total países & Argentina & Brasil & Chile & Colombia & México & Perú & Uruguay \\
\hline \multicolumn{9}{|l|}{ Matemáticas } \\
\hline $\begin{array}{l}\Delta \text { (brecha bruta }) \\
\Delta_{0}(\text { brecha neta })\end{array}$ & $15,0(0,05)$ & $20,5(0,59)$ & $13,9(0,15)$ & $12,3(0,19)$ & $13,0(0,24)$ & $13,8(0,13)$ & $14,2(0,24)$ & $18,7(0,23)$ \\
\hline \multicolumn{9}{|l|}{ Controles } \\
\hline Localización urbana & 13,2 & 18,7 & 12,7 & 11,0 & 9,6 & 11,9 & 8,4 & 17,1 \\
\hline +Escuela pública & 11,3 & 15,0 & 8,9 & 8,8 & 7,6 & 11,6 & 7,5 & 14,3 \\
\hline +Familia nuclear & 10,7 & 14,9 & 8,5 & 8,8 & 7,1 & 10,9 & 7,4 & 13,2 \\
\hline +NSE hogar & 7,8 & 9,9 & 6,4 & 4,0 & 2,6 & 8,2 & 4,6 & 9,2 \\
\hline +Nota media pares & $3,9(0,04)$ & $6,1(0,45)$ & $4,6(0,14)$ & $3,6(0,21)$ & $1,5(0,26)$ & $5,1(0,11)$ & $3,0(0,19)$ & $6,9(0,20)$ \\
\hline
\end{tabular}

Lectura

\begin{tabular}{|c|c|c|c|c|c|c|c|c|}
\hline$\Delta$ (brecha bruta) & $14,5(0,06)$ & $25,4(0,77)$ & $15,8(0,16)$ & $11,2(0,19)$ & $12,8(0,26)$ & $14,8(0,17)$ & $16,2(0,28)$ & $20,6(0,28)$ \\
\hline$\Delta_{0}($ brecha neta $)$ & & & & & & & & \\
\hline \multicolumn{9}{|l|}{ Controles } \\
\hline Localización urbana & 12,7 & 23,3 & 14,4 & 10,0 & 9,3 & 12,7 & 10,0 & 18,8 \\
\hline +Escuela pública & 10,7 & 18,7 & 10,8 & 7,8 & 7,3 & 12,4 & 9,1 & 15,9 \\
\hline +Familia nuclear & 10,2 & 18,7 & 10,4 & 7,6 & 6,8 & 11,7 & 9,1 & 14,8 \\
\hline +NSE hogar & 7,1 & 13,5 & 7,9 & 3,2 & 2,1 & 8,7 & 5,9 & 10,6 \\
\hline+ Nota media pares & $4,2(0,05)$ & $7,9(0,53)$ & $5,8(0,15)$ & $2,9(0,21)$ & $1,5(0,25)$ & $4,9(0,13)$ & $3,6(0,21)$ & $8,6(0,21)$ \\
\hline
\end{tabular}

"Asistió 1 año o menos" comparado con "No asistió"

\begin{tabular}{|c|c|c|c|c|c|c|c|c|}
\hline & Total países & Argentina & Brasil & Chile & Colombia & México & Perú & Uruguay \\
\hline \multicolumn{9}{|l|}{ Matemáticas } \\
\hline $\begin{array}{l}\Delta \text { (brecha bruta) } \\
\Delta_{0}(\text { brecha neta })\end{array}$ & $8,9(0,05)$ & $10,4(0,53)$ & $7,9(0,15)$ & $6,9(0,16)$ & $10,6(0,20)$ & $10,9(0,14)$ & $8,3(0,26)$ & $10,1(0,29)$ \\
\hline \multicolumn{9}{|l|}{ Controles } \\
\hline Localización urbana & 8,2 & 9,5 & 7,6 & 6,2 & 9,3 & 9,4 & 5,8 & 9,8 \\
\hline +Escuela pública & 7,1 & 9,6 & 6,1 & 4,9 & 8,6 & 9,4 & 5,4 & 9,4 \\
\hline +Familia nuclear & 6,6 & 9,5 & 5,4 & 4,7 & 8,1 & 8,6 & 5,4 & 8,4 \\
\hline +NSE hogar & 5,2 & 8,4 & 4,6 & 2,6 & 5,8 & 7,9 & 3,3 & 7,7 \\
\hline +Nota media pares & $3,1(0,04)$ & $7,5(0,46)$ & $2,8(0,12)$ & $1,6(0,16)$ & $4,6(0,21)$ & $4,2(0,12)$ & $2,1(0,23)$ & $6,5(0,28)$ \\
\hline
\end{tabular}

Lectura

\begin{tabular}{|c|c|c|c|c|c|c|c|c|}
\hline $\begin{array}{l}\Delta(\text { brecha bruta }) \\
\Delta_{0}(\text { brecha neta })\end{array}$ & $9,9(0,06)$ & $14,3(0,75)$ & $9,3(0,15)$ & $8,1(0,17)$ & $10,2(0,26)$ & $12,2(0,17)$ & $9,2(0,32)$ & $9,9(0,34)$ \\
\hline \multicolumn{9}{|l|}{ Controles } \\
\hline Localización urbana & 9,1 & 13,1 & 8,9 & 7,5 & 8,6 & 10,3 & 6,3 & 9,5 \\
\hline +Escuela pública & 8,0 & 13,2 & 7,5 & 6,0 & 7,9 & 10,3 & 5,8 & 9,1 \\
\hline +Familia nuclear & 7,4 & 13,2 & 6,9 & 5,8 & 7,4 & 9,4 & 5,8 & 8,0 \\
\hline +NSE hogar & 5,9 & 12,1 & 6,0 & 4,0 & 5,2 & 8,5 & 3,3 & 7,5 \\
\hline+ Nota media pares & $3,5(0,05)$ & $7,9(0,59)$ & $4,0(0,13)$ & $3,1(0,18)$ & $3,3(0,24)$ & $3,9(0,15)$ & $1,3(0,24)$ & $6,5(0,30)$ \\
\hline umnos) & $88683(7,7)$ & $4774(4,6)$ & $20127(9,0)$ & $5669(13,2)$ & $7921 \quad(9,1)$ & $38250(7,2)$ & $5985(1,9)$ & $5957(3,7)$ \\
\hline
\end{tabular}

Fuente: Elaboración propia sobre la base de Organización para la Cooperación y el Desarrollo Económicos (ocDE), PISA 2009 Technical Report, París, OECD Publishing, 2012.

Nota: Las diferencias en las brechas brutas y netas entre los distintos países resultan estadísticamente significativas al nivel del $1 \%$ para todos los casos, utilizando una técnica de bootstrap de 200 réplicas; tanto para la brecha bruta $\left(\Delta\right.$, ecuación (1)) como para $\Delta_{0}$ final (brecha neta luego de todos los controles, ecuación (7)) se presenta entre paréntesis el error estándar de la brecha; $\mathrm{N}$ indica el tamaño de la muestra y entre paréntesis se detalla el porcentaje de observaciones perdidas al incluir todos los controles. NSE corresponde al nivel socioeconómico del hogar. 
CUADRO 5

Descomposición de las brechas de puntajes en las pruebas PISA, 2012

(En porcentajes)

\begin{tabular}{|c|c|c|c|c|c|c|c|c|}
\hline & Total países & Argentina & Brasil & Chile & Colombia & México & Perú & Uruguay \\
\hline \multicolumn{9}{|l|}{ Matemáticas } \\
\hline $\begin{array}{l}\Delta \text { (brecha bruta) } \\
\left.\Delta_{0} \text { (brecha neta }\right)\end{array}$ & $12,3(0,08)$ & $19,5(0,38)$ & $10,8(0,11)$ & $14,2(0,23)$ & $9,8(0,23)$ & $10,9(0,12)$ & $17,1(0,25)$ & $15,3(0,21)$ \\
\hline \multicolumn{9}{|l|}{ Controles } \\
\hline Localización urbana & 11,1 & 19,0 & 9,4 & 12,4 & 8,3 & 9,3 & 13,3 & 13,3 \\
\hline +Escuela pública & 9,4 & 16,4 & 7,0 & 9,4 & 6,5 & 8,9 & 10,1 & 10,6 \\
\hline +Familia nuclear & 8,6 & 15,4 & 6,2 & 8,8 & 6,1 & 8,0 & 9,7 & 9,3 \\
\hline +NSE hogar & 6,4 & 11,9 & 4,7 & 3,0 & 2,3 & 6,0 & 5,8 & 6,9 \\
\hline +Nota media pares & $3,3(0,06)$ & $8,1(0,33)$ & $3,1(0,09)$ & $0,7(0,23)$ & $0,9(0,24)$ & $3,7(0,09)$ & $3,8(0,22)$ & $4,6(0,18)$ \\
\hline
\end{tabular}

Lectura

\begin{tabular}{|c|c|c|c|c|c|c|c|c|}
\hline $\begin{array}{l}\Delta \text { (brecha bruta) } \\
\Delta_{0} \text { (brecha neta) }\end{array}$ & $12,8(0,08)$ & $22,4(0,51)$ & $12,4(0,15)$ & $12,7(0,23)$ & $10,4(0,27)$ & $12,2(0,13)$ & $17,4(0,23)$ & $17,0(0,27)$ \\
\hline \multicolumn{9}{|l|}{ Controles } \\
\hline Localización urbana & 11,4 & 21,7 & 11,0 & 10,6 & 8,8 & 10,2 & 12,9 & 14,6 \\
\hline +Escuela pública & 9,8 & 18,4 & 8,8 & 8,0 & 6,8 & 9,8 & 10,1 & 12,0 \\
\hline +Familia nuclear & 9,0 & 17,4 & 8,0 & 7,4 & 6,4 & 8,9 & 9,8 & 10,7 \\
\hline +NSE hogar & 6,7 & 14,1 & 6,8 & 2,4 & 2,4 & 6,7 & 5,5 & 8,7 \\
\hline +Nota media pares & $3,9(0,06)$ & $10,6(0,52)$ & $4,5(0,12)$ & $0,6(0,24)$ & $0,1(0,29)$ & $4,7(0,12)$ & $2,3(0,24)$ & $6,6(0,26)$ \\
\hline
\end{tabular}

"Asistió 1 año o menos" comparado con "No asistió"

\begin{tabular}{|c|c|c|c|c|c|c|c|c|}
\hline & Total países & Argentina & Brasil & Chile & Colombia & México & Perú & Uruguay \\
\hline \multicolumn{9}{|l|}{ Matemática } \\
\hline $\begin{array}{l}\Delta \text { (brecha bruta) } \\
\Delta_{0} \text { (brecha neta) }\end{array}$ & $6,7(0,08)$ & $8,5(0,35)$ & $4,7(0,12)$ & $10,8(0,22)$ & $8,3(0,21)$ & $8,8(0,14)$ & $10,1(0,24)$ & $5,5(0,26)$ \\
\hline \multicolumn{9}{|l|}{ Controles } \\
\hline Localización urbana & 6,0 & 8,0 & 4,4 & 10,4 & 7,3 & 7,6 & 7,9 & 4,9 \\
\hline +Escuela pública & 5,2 & 8,5 & 3,6 & 8,8 & 7,2 & 7,5 & 6,5 & 3,9 \\
\hline +Familia nuclear & 4,4 & 7,5 & 2,8 & 8,0 & 6,4 & 6,4 & 6,1 & 3,3 \\
\hline +NSE hogar & 3,4 & 6,8 & 2,6 & 4,4 & 4,1 & 5,6 & 3,3 & 2,7 \\
\hline +Nota media pares & $1,9(0,06)$ & $3,2(0,44)$ & $0,8(0,11)$ & $2,6(0,23)$ & $2,2(0,21)$ & $2,9(0,13)$ & $1,7(0,28)$ & $0,68(0,26)$ \\
\hline
\end{tabular}

Lectura

\begin{tabular}{lllllllll}
\hline$\Delta$ (brecha bruta) & $7,8(0,09)$ & $10,7(0,44)$ & $5,8(0,15)$ & $10,3(0,21)$ & $8,6(0,24)$ & $10,2(0,14)$ & $9,4(0,27)$ & $7,7(0,32)$ \\
$\quad \Delta_{0}$ (brecha neta) & & & & & & & & \\
Controles & & & & & & & \\
Localización urbana & 7,0 & 10,1 & 5,5 & 9,5 & 7,4 & 8,6 & 7,0 & 6,9 \\
$\quad$ +Escuela pública & 6,1 & 10,8 & 4,8 & 8,0 & 7,2 & 8,5 & 5,8 & 5,9 \\
+Familia nuclear & 5,4 & 10,0 & 3,9 & 7,2 & 6,5 & 7,4 & 5,5 & 5,4 \\
$\quad$ +NSE hogar & 4,5 & 8,7 & 3,2 & 3,8 & 3,9 & 6,6 & 2,8 & 5,0 \\
+Nota media pares & $2,3(0,07)$ & $7,7(0,53)$ & $1,3(0,14)$ & $2,1(0,22)$ & $2,7(0,25)$ & $4,0(0,14)$ & $0,15(0,29)$ & $3,5(0,30)$ \\
\hline
\end{tabular}

N (número de alumnos) $86197(18,9) 5908(20,4) 19204(18,1) 6856(14,5) 9073(26,5) 33806(19,0) 6035(13,6) 5315(18,6)$

Fuente: Elaboración propia sobre la base de Organización para la Cooperación y el Desarrollo Económicos (OCDE), PISA 2009 Technical Report, París, OECD Publishing, 2012.

Nota: Las diferencias en las brechas brutas y netas entre los distintos países resultan estadísticamente significativas al nivel del $1 \%$ para todos los casos, utilizando una técnica de bootstrap de 200 réplicas; tanto para la brecha bruta $\left(\Delta\right.$, ecuación (1)) como para $\Delta_{0}$ final (brecha neta luego de todos los controles, ecuación (7)) se presenta entre paréntesis el error estándar de la brecha; $\mathrm{N}$ indica el tamaño de la muestra, y entre paréntesis se detalla el porcentaje de observaciones perdidas al incluir todos los controles. NSE corresponde al nivel socioeconómico del hogar. 
más de un año y quienes no lo hacen en la Argentina en 2012 se explica por otros factores. Esta diferencia de unos 36 puntos es ciertamente relevante, ya que representa a su vez un $9 \%$ del puntaje promedio para todos los jóvenes participantes del país, y casi la mitad de su desvío estándar.

En el otro extremo, Chile, Colombia y el Perú en algunos casos, presentan las menores diferencias. Para ejemplificar, en 2009 Chile registra la mínima brecha $(1,6 \%)$ en el área de Matemáticas, para una asistencia menor o igual a un año. Esto representa unos seis puntos, es decir, aproximadamente 1,5\% del puntaje promedio para el país en esta competencia. Estas diferencias en las brechas no explicadas permiten suponer que existen efectos heterogéneos no observables entre países que se traducen en brechas finales distintas en los resultados cognitivos futuros.

En cuanto al grado de exposición al "tratamiento" (un año o menos comparado con más de un año), los resultados son consistentes con lo esperado: las brechas brutas son mayores con períodos de asistencia al preescolar más prolongados. Esto podría deberse tanto al efecto propio de haber recibido por más tiempo una educación temprana, como al efecto de los factores socioeconómicos que inciden tanto en la permanencia en el preescolar como en los resultados de las pruebas de PISA.

Con las excepciones de Colombia y Chile, esta relación se mantiene al controlar por dichas influencias, evaluando las brechas netas $\left(\Delta_{0}\right.$, ecuación (7)). Por ejemplo, con respecto al año 2012 y considerando la muestra conjunta de países, los niños que asistieron

\section{V}

\section{Conclusiones}

América Latina tiene un panorama heterogéneo tanto en la cobertura de la educación inicial como en sus avances durante las últimas décadas. Impulsados por un consenso político y académico, los compromisos internacionales asumidos se han plasmado en una serie de políticas tendientes a expandir el acceso al preescolar. Así, la obligatoriedad de este nivel se estableció en la mayoría de los países de la región desde fines de los años noventa, contribuyendo al incremento de las tasas de asistencia escolar entre los más pequeños. durante más de un año al preescolar obtuvieron un puntaje promedio en Matemáticas que superaba en un 3,3\% al de quienes no habían accedido a este nivel; mientras que aquellos que solo habían asistido un año o menos obtuvieron resultados superiores en un 1,9\% a los de este último grupo. Esto refuerza la noción de que no solo importa el acceso al nivel inicial, sino también la duración de la asistencia, lo que sugiere la conveniencia de fomentar la inclusión a edades más tempranas.

Con respecto a la evolución de los resultados entre ambas rondas de PISA, puede afirmarse que, con algunas excepciones, las brechas brutas y netas se han reducido. Esto podría estar evidenciando que el aumento de los programas vinculados a la educación inicial desde finales de los años noventa en la región (período en que estas cohortes asistieron al preescolar) contribuyó a la reducción de las desigualdades de acceso y, por lo tanto, se tienen brechas inferiores. Por otra parte, esto podría derivarse de una menor incidencia de la asistencia al nivel inicial en el rendimiento académico. Sin embargo, dado que el lapso de tiempo transcurrido entre ambas evaluaciones es relativamente corto, resulta aventurado plantear una hipótesis en cualquiera de estas direcciones.

Por último, la comparación de las brechas explicadas y no explicadas entre las dos competencias evaluadas en PISA sugiere que el efecto de asistir al preescolar y de sus factores asociados es, en general, mayor para el área de Lectura que para Matemáticas, lo que es un indicio de la importancia de la interacción con personas de la misma edad para el desarrollo del lenguaje en esas etapas de la vida.
No obstante, aún permanecen excluidos de la educación inicial niños provenientes de contextos más desfavorecidos, residentes de zonas rurales, con un bajo nivel socioeconómico familiar, o ambos casos. La revisión de estas desigualdades de acceso resulta prioritaria para cualquier política de equidad, aun cuando las diferencias se han venido reduciendo lentamente. El análisis realizado sugiere que los diferenciales de puntajes inicialmente estimados disminuyen al incluir los distintos atributos escogidos. Sin embargo, no todos los factores parecen 
tener la misma capacidad de explicar las brechas: las variables que reflejan el nivel socioeconómico de los hogares y la calidad de la escuela secundaria reducen en mayor medida el componente no explicado, frente a la estructura familiar o el área de residencia.

Ante la escasez de investigaciones empíricas sobre el tema en la región, mediante el presente análisis se aporta evidencia sobre la influencia de la educación temprana en los logros educativos futuros. La principal conclusión es que, aun controlando por variables fundamentales del contexto del estudiante, en muchos casos el componente no explicado de las brechas de rendimiento constituye un porcentaje considerable de la brecha total y, por lo tanto, representa un diferencial de puntaje significativo. No se puede hablar de un estudio de efecto causal, porque no existió una asignación aleatoria de los estudiantes a grupos de tratamiento (asistir al preescolar) o control (no hacerlo) que garantice la eliminación de sesgos en las estimaciones. Este tipo de ejercicios es altamente costoso y no hay iniciativas previas que permitan su aplicación. Sin embargo, los controles empleados en la construcción del emparejamiento reducen el efecto de los factores observables y no observables que tenderían a sesgar el análisis. Se han incluido los principales atributos que determinan el acceso al preescolar y que, según la literatura especializada, inciden en el rendimiento académico en el nivel medio. Cabe destacar, además, que aceptando que las poblaciones más vulnerables tienen menos probabilidades de acceder al preescolar y más probabilidades de abandonar el sistema, los efectos hallados subestiman el tamaño del efecto, es decir, son solo un límite inferior del verdadero efecto, pero el resultado va en la misma dirección.
Los resultados obtenidos tienen una clara implicancia política. En primer lugar, refuerzan los argumentos en favor de universalizar el acceso a la educación inicial en la región y monitorear las diferencias en calidad, principalmente en los entornos de mayor vulnerabilidad. Tanto por el valor instrumental del preescolar aquí analizado, como por su valor intrínseco, es importante continuar generando iniciativas que permitan la inclusión de la población de niños más vulnerables y su permanencia en el sistema educativo.

En segundo lugar, las diferencias entre países en la magnitud de las brechas de puntajes sugieren analizar el papel que juegan otros factores como los arreglos institucionales, las características de los mercados educativos y los compromisos presupuestarios. El efecto del preescolar se diluye en algunos sistemas más que en otros luego del paso por la escuela primaria y secundaria, quizás como resultado de diferencias importantes en la calidad de los programas ofrecidos entre los sistemas públicos y privados de provisión de educación. Esto implica poner un mayor énfasis en el mejoramiento, la homogenización y la evaluación de las distintas ofertas en este nivel educativo. Finalmente, se encontró que no solo importa la asistencia al preescolar, sino también su duración, lo que va en la misma línea de los resultados de Barnett (1995) y Reynolds (1995). Por lo tanto, cobran relevancia los reclamos porque los esfuerzos y las políticas se orienten a extender la cobertura en las edades más tempranas.

En conclusión, los compromisos de los países latinoamericanos aún dejan pendientes desafíos relevantes por enfrentar, apreciándose una brecha entre las expresiones de deseo plasmadas en la legislación y los resultados reales.

\section{Bibliografía}

Adams, R. y M. Wu (eds.) (2002), PISA 2000 Technical Report, París, OECD Publishing.

Albergucci, M. (2006), Educación inicial. Análisis cuantitativo del nivel, Buenos Aires, Ministerio de Educación, Ciencia y Tecnología de la Nación.

Arteaga, I. y otros (2014), "One year of preschool or two: is it important for adult outcomes?", Economics of Education Review, vol. 40, Amsterdam, Elsevier.

Barnett, S. (1995), "Long-term effects of the early childhood programs on cognitive and school outcomes", The Future of Children, vol. 5, $\mathrm{N}^{\circ} 3$, Princeton, Princeton-Brookings.

Barnett, S. y C. Lamy (2006), Estimated Impacts of Number of Years of Preschool Attendance on Vocabulary, Literacy and Math Skills at Kindergarten Entry, New Brunswick, National Institute for Early Education Research.

Berhman, J., Y. Cheng y P. Todd (2004), "Evaluating preschool programs when length of exposure to the program varies: a nonparametric approach", The Review of Economics and
Statistics, vol. 86, $\mathrm{N}^{\circ} 1$, Cambridge, Massachusetts, The MIT Press.

Blinder, A. (1973), "Wage discrimination: reduced form and structural estimates", The Journal of Human Resources, vol. 7, $\mathrm{N}^{\circ} 4$, Madison, University of Wisconsin Press.

Burchinal, M. y otros (1997), "Early intervention and mediating processes in cognitive performance of children of low-income African-American families", Child Development, vol. 68, $\mathrm{N}^{\circ}$ 5, Wiley.

Calero, J. y otros (2007), "Desigualdades socioeconómicas en el sistema educativo español", Investigación, $\mathrm{N}^{\circ} 176$, Madrid.

Camilli, G. y otros (2010), "Meta-analysis of the effects of early education interventions on cognitive and social development", Teachers College Record, vol. 112, N 3, Nueva York, Teachers College.

CEPAL (Comisión Económica para América Latina y el Caribe) (2008), Panorama Social de América Latina 2007 (LC/ G.2351-P), Santiago. 
Cordero, T. (2004), "Educación inicial en América Latina: situaciones y retos. Caso panameño", Educación, vol. 28, № 1, San José, Universidad de Costa Rica.

Cunha, F. y otros (2006), "Interpreting the evidence on life cycle skill formation", Handbook of the Economics of Education, E. Hanushek y F. Welch (eds.), Amsterdam, North-Holland.

Currie, J. (2001), "Early childhood intervention programs: what do we know?", Journal of Economic Perspectives, vol. 15, ํㅜㄹ, Nashville, Tennessee, American Economic Association.

Diker, G. (2003), Organización y perspectivas de la educación inicial en Iberoamérica: principales tendencias, Organización de Estados Iberoamericanos para la Educación, la Ciencia y la Cultura (OEI) [en línea] www.oei.es/linea3/diker.pdf.

Formichella, M. y N. Krüger (2013), "El fracaso escolar en el nivel medio argentino: ¿es menos frecuente en las escuelas de gestión privada debido a su administración?", Regional and Sectoral Economic Studies, vol. 13, $\mathrm{N}^{\circ} 3$, Asociación de Estudios Euroamericanos de Desarrollo Económico (AEEADE).

Glewwe, P. y H. Jacoby (1995), "An economic analysis of delayed primary school enrollment in a low income country: the role of early childhood nutrition", The Review of Economics and Statistics, vol. 77, $\mathrm{N}^{\circ}$ 1, Cambridge, Massachusetts, The MIT Press.

Hazarika, G. y V. Viren (2013), "The effect of early childhood developmental program attendance on future school enrollment in rural North India", Economics of Education Review, vol. 34, Amsterdam, Elsevier.

Heckman, J. (2008), "Schools, skills and synapses", NBER Working Papers, $\mathrm{N}^{\circ} 14064$, Cambridge, Massachusetts, National Bureau of Economic Research (NBER).

(2000), "Policies to foster human capital", Research in Economics, vol. 54, $\mathrm{N}^{\circ} 1$, Amsterdam, Elsevier.

Krüger, N. (2013), "Segregación social y desigualdad de logros educativos en Argentina", Archivos analíticos de políticas educativas, vol. 21, $\mathrm{N}^{\circ} 86$, Tucson, Arizona State University.

Krüger, N., M. Formichella y A. Lekuona (2015), "Más allá de los logros cognitivos: la actitud hacia la escuela y sus determinantes en España según PISA 2009”, Revista de Educación, vol. 367, Madrid, Ministerio de Educación, Cultura y Deporte.

Magnuson, K., C. Ruhm y J. Waldfogel (2007a), "Does prekindergarten improve school preparation and performance?", Economics of Education Review, vol. 26, $\mathrm{N}^{\circ}$ 1, Amsterdam, Elsevier.

(2007b), "The persistence of preschool effects: do subsequent classroom experience matter?", Early Childhood Research Quarterly, vol. 22, № 1, Amsterdam, Elsevier.
MEC (Ministerio de Educación de Brasil) (2013), La educación infantil en los países del MERCOSUR: un análisis comparativo de la legislación, Brasilia.

Mizala, A. y H. Ñopo (2012), "Evolution of teachers' salaries in Latin America at the turn of the 20th century: how much are they (under or over) paid?", IZA Discussion Papers, $\mathrm{N}^{\circ}$ 6806, Bonn, Institute for the Study of Labor (IZA).

Nores, M. y S. Barnett (2010), "Benefits of early childhood interventions across the world: (under) investing in the very young", Economics of Education Review, vol. 29, $\mathrm{N}^{\circ} 2$, Amsterdam, Elsevier.

Ñopo, H. (2008), "Matching as a tool to decompose wage gaps", The Review of Economics and Statistics, vol. 90, N², Cambridge, Massachusetts, The MIT Press.

Oaxaca, R. (1973), "Male-female wage differentials in urban labor market", International Economic Review, vol. 14, $\mathrm{N}^{\circ} 3$, Wiley.

OCDE (Organización para la Cooperación y el Desarrollo Económicos) (2014), PISA 2012 Results: What Students Know and Can Do: Student Performance in Mathematics, Reading and Science (Volume 1), París, OECD Publishing.

(2013), PISA 2012 Results: Ready to Learn: Students' Engagement, Drive and Self-Beliefs (Volume III), París, OECD Publishing.

(2012), PISA 2009 Technical Report, París, OECD Publishing.

Reynolds, A. (1995), "One year of preschool intervention or two: does it matter?", Early Childhood Research Quarterly, vol. 10, $\mathrm{N}^{\circ} 1$, Amsterdam, Elsevier.

Ritblatt, S. y otros (2001), "Are two better than one? The impact of years in head start on child outcomes, family environment, and reading at home", Early Childhood Research Quarterly, vol. 16, $\mathrm{N}^{\circ} 4$, Amsterdam, Elsevier.

siteal (Sistema de Información sobre la Primera Infancia en América Latina) (2013), "Conversación con Margarita Poggi. La agenda educativa latinoamericana", Buenos Aires [en línea] http://www.siteal.iipe-oei.org/debates/427/dialogo-conmargarita-poggi.

(2010), Sistema de información sobre los derechos del niño en la primera infancia en los países de América Latina. Marco teórico y metodológico, Buenos Aires.

(2009), Primera infancia en América Latina: la situación actual y las respuestas desde el Estado. Informe sobre tendencias sociales y educativas en América Latina 2009, Buenos Aires.

Skibbe, L. y otros (2011), "Schooling effects on preschoolers' self-regulation, early literacy, and language growth", Early Childhood Research Quarterly, vol. 26, $\mathrm{N}^{\circ}$ 1, Amsterdam, Elsevier. 